\title{
Classification of Steiner quadruple systems of order 16 and rank $14 .^{*}$
}

\author{
V. A. Zinoviev and D. V. Zinoviev \\ Institute for Problems of Information Transmission \\ Russian Academy of Sciences \\ Bol'shoi Karetnyi, 19, GSP-4, Moscow, 101447, Russia
}

\begin{abstract}
A Steiner quadruple system $S(v, 4,3)$ of order $v$ is a 3 -design $T(v, 4,3, \lambda)$ with $\lambda=1$. In the previous paper [1] we classified all such Steiner systems $S(16,4,3)$ of order 16 with rank 13 or less over $\mathbb{F}_{2}$. In particular, we have proved that there is one $S(16,4,3)$ of rank 11 (the points and planes of affine geometry AG(4,2)), fifteen systems $S(16,4,3)$ of rank 12 and 4131 systems of rank 13 . In this paper we describe all non-isomorphic $S(16,4,3)$ of rank 14 over $\mathbb{F}_{2}$. All these Steiner systems $S(16,4,3)$ can be obtained by the generalized doubling construction, which we give here. Our main result is that there are exactly 684764 non-isomorphic Steiner quadruple systems $S(16,4,3)$ of order 16 with rank 14 . We found all non-isomorphic homogenious systems with rank 14 over $\mathbb{F}_{2}$.
\end{abstract}

*The paper has been written under the partial financial support of the Russian fund for the fundamental research (the number of project $03-01-00098$ ) 


\section{$\S 1$. Introduction}

A Stener system $S(n, k, t)$ is a pair $(X, B)$ where $X$ is a $v$-set and $B$ is a collection of $k$-subsets of $X$ such that every $t$-subset of $X$ is contained in exactly one member of $B$.

A system $S(v, 3,2)$ is called a Steiner triple system (briefly $\operatorname{STS}(v)$ ) and a system $S(v, 4,3)$ is called a Steiner quadruple system (briefly $\operatorname{SQS}(v)$ ). The necessary condition for existence of an $\operatorname{SQS}(v)$ is that $v \equiv 2$ or $4(\bmod 6)$. Hanani $[2]$ proved that the necessary condition $v \equiv 2$ or $4(\bmod 6)$ for the existence of an $S(v, 4,3)$ is also sufficient.

Two systems $\operatorname{SQS}(X, B)$ and $\operatorname{SQS}\left(X^{\prime}, B^{\prime}\right)$ are isomorphic, if there is a bijection $\alpha: X \rightarrow$ $X^{\prime}$ that maps the quadruples of $B$ to those of $B^{\prime}$. An automorphism of $\operatorname{SQS}(X, B)$ is an isomorphism of $(X, B)$ to itself. The determination of number of the non-isomorphic $\operatorname{SQS}(v)$, which we will denote by $N(v)$, is the major problem in this area. Barrau [3] proved that $N(v)=1$ for $v \leq 10$ and Mendelson and Hung [4] derived with the help of a computer that $N(14)=4$.

In [5] it was shown that $N(16) \geq 8$. Using computer assisted computations, Gibbons, Mathon and Corneil [6] proved that $N(16) \geq 282$. The knowledge of all non-isomorphic 1-factorizations of $K_{8}$ (the complete graph on 8 vertices) together with their automorphism groups allowed Lindner and Rosa [7], using the classical doubling construction, obtained the bound $N(16) \geq 31021$ (for the number of systems with rank exactly 14 over $\mathbb{F}_{2}$ ). They slightly improved this bound in [8]: $N(16) \geq 31301$ (adding systems with rank less or equal 13 over $\mathbb{F}_{2}$ ). No progress has been made in this regard since this result of Lindner and Rosa (see [9], [10]).

Our result of [1] can be formulated as follows. Among the non-isomorphic Steiner systems $S(16,4,3)$ of order $v=16$ there are:

- one $S(16,4,3)$ of rank 11 (the points and planes of 4-dimensional affine geometry $A G(4,2)$ over $\left.\mathbb{F}_{2}\right)$;

- 15 systems $S(16,4,3)$ of rank 12 ;

- 4131 systems $S(16,4,3)$ of rank 13 .

This paper is a natural continuation of our previous paper [1] where we started the systematic investigation of Steiner systems $S(16,4,3)$ of order 16 with given rank over the field $\mathbb{F}_{2}$. Here we classified all Steiner systems $S(16,4,3)$ of order 16 with rank 14 over the field $\mathbb{F}_{2}$. All such systems can be obtained by the generalized doubling construction, which we introduce here.

Our main result here can be formulated as follows. Among the non-isomorphic Steiner systems $S(16,4,3)$ of order $v=16$ there are:

- 684764 systems $S(16,4,3)$ of rank 14 over $\mathbb{F}_{2}$.

The paper is organized as follows. Preliminary results and terminology are given in $\S 2$. In $\S 3$ we describe the classical doubling construction of $\operatorname{SQS}(2 n)$ using given $\operatorname{SQS}(n)$. In $\S 4$ we consider the general properties of $\operatorname{SQS}(n)$ with rank $n-2$ over $\mathbb{F}_{2}$. Section $\S 5$ is dedicated to the generalized doubling construction of Steiner systems $S(n, 4,3)$ of arbitrary order $n$. The paragraph $\S 6$ contains the main result of the paper: classification of all non-isomorphic Steiner systems $S(16,4,3)$ with rank 14 over $\mathbb{F}_{2}$. In $\S 7$ we give some results concerning the Steiner triple systems $S(15,3,2)$ which occur as derivative of all these non-isomorphic 
$S(16,4,3)$ with rank 14 . In particular, we found only such triple systems with numbers $1,2, \ldots, 22$ and 62 . We also found all homogeneous Steiner systems $S(16,4,3)$ of rank 14 (and from [1] we know such systems with ranks 11, 12 and 13). We give also the distribution of the number $\beta$ (the number of non-somorphic derivative $S(15,3,2)$ of given $S(16,4,3)$ ) over all these systems $S(16,4,3)$ with rank 14 .

\section{$\S 2$. Preliminary results}

Let $E$ be a binary alphabet of size 2: $E=\{0,1\}$. A binary code of length $n$ is an arbitrary subset of $E^{n}$. Denote such binary code $C$ with length $n$, with the minimal distance $d$ and cardinality $N$ as $(n, d, N)$-code. Denote by wt $(\boldsymbol{x})$ the Hamming weight of vector $\boldsymbol{x}$ over $E$. For a (binary) code $C$ denote by $\langle C\rangle$ the linear envelope of words of $C$ over $\mathbb{F}_{2}$. The dimension of space $\langle C\rangle$ is called the rank of $C$ over $\mathbb{F}_{2}$ and is denoted $\operatorname{rank}(C)$.

Denote by $(n, w, d, N)$ a binary constant weight code $W$ of length $n$, with weight of all codewords $w$, with minimal distance $d$ and cardinality $N$.

For any two subsets $Y$ and $Z$ of $E^{n}$ denote by $d(Y, Z)$ the minimal distance between $Y$ and $Z$ :

$$
d(Y, Z)=\min \{d(\boldsymbol{y}, \boldsymbol{z}): \boldsymbol{y} \in Y, \boldsymbol{z} \in Z\} .
$$

For vector $\boldsymbol{v}=\left(v_{1}, \ldots, v_{n}\right) \in E^{n}$ denote by $\operatorname{supp}(\boldsymbol{v})$ its support, i.e. the set of indices with nonzero positions:

$$
\operatorname{supp}(\boldsymbol{v})=\left\{i: v_{i} \neq 0\right\} .
$$

Denote by $\overline{\boldsymbol{v}}$ a vector, which is a complementary to $\boldsymbol{v}$, i.e. $\bar{v}_{i}=v_{i}+\mathbf{1}$.

If $E=\mathbb{F}_{2}$ is a field of order 2 , the binary $(n, d, N)$-code $A$ which is a linear $k$-dimensional space over $\mathbb{F}_{q}$ is denoted by $[n, k, d]$-code. For binary vectors $\boldsymbol{x}=\left(x_{1}, \cdots, x_{n}\right)$ and $\boldsymbol{y}=$ $\left(y_{1}, \cdots, y_{n}\right)$ denote by $(\boldsymbol{x} \cdot \boldsymbol{y})=x_{1} y_{1}+\cdots+x_{n} y_{n}$ their inner product over $\mathbb{F}_{2}$. For a linear $[n, k, d]$-code $A$ denote by $A^{\perp}$ its dual code:

$$
A^{\perp}=\left\{\boldsymbol{v} \in \mathbb{F}_{2}^{n}:(\boldsymbol{v} \cdot \boldsymbol{c})=0, \forall \boldsymbol{c} \in A\right\} .
$$

It is clear that $A^{\perp}$ is a linear $\left[n, n-k, d^{\perp}\right]$ code with some minimal distance $d^{\perp}$.

Denote by $E_{2}^{n}$ the set of all binary vectors of length $n$ of weight 2 . Let $J_{n}=\{1,2, \ldots, n\}$ be the coordinate set of $E^{n}$ and let $S_{n}$ be the full group of permutations of $n$ elements. For any $i \in J_{n}$ and $\pi \in S_{n}$, define the image of $i$ under the action of $\pi$ by $\pi(i)$. For any set $X$ of $E^{n}$ and any $\pi \in S_{n}$ denote $\pi X=\{\pi(\boldsymbol{x}): \boldsymbol{x} \in X\}$.

A binary incidence matrix of a Steiner system $S(v, 4,3)$ is the binary constant weight code $(v, 4,4, v(v-1)(v-2) / 24)$, denoted by $S$ which is strongly optimal [15]. In our notation the connection between the system $(X, B)$ and the code $S$ looks as follows:

$$
B=\{\operatorname{supp}(\boldsymbol{v}) \subset X: \boldsymbol{v} \in S\} .
$$

For any Steiner system $S(v, 4,3)$ denote by $\mu_{s}(\boldsymbol{c})$, where $\boldsymbol{c} \in S$ and $s \in\{0,1,2\}$, the number of codewords $\boldsymbol{x} \in S$ with distance $2(k-s)$ at $\boldsymbol{c}$, i.e.

$$
\mu_{s}(\boldsymbol{c})=|\{\boldsymbol{x} \in S:|\operatorname{supp}(\boldsymbol{c}) \cap \operatorname{supp}(\boldsymbol{x})|=s\}|, \quad s \in\{0,1,2\} .
$$


The numbers $\mu_{s}(\boldsymbol{c})$ do not depend on the choice of $\boldsymbol{c}$ and can be computed explicitly (see Theorem 5 in [15]). In particular, for $S(16,4,3)$ we have:

$$
\mu_{0}=39, \quad \mu_{1}=64, \quad \mu_{2}=36
$$

For the case of Steiner systems the definition of equivalence can be formulated as follows.

Definition 1 Two Steiner systems $(X, B)$ and $\left(X^{\prime}, B^{\prime}\right)$ of order 16 are isomorphic, if their incidence matrices $S$ and $S^{\prime}$ are equivalent as constant weight codes, i.e. if there exists some permutation $\tau \in S_{16}$ such that $S$ and $\tau S^{\prime}$ coincide up to the permutation of rows.

\section{$\S 3$. SQS $(2 n)$ obtained by the doubling construction from $\operatorname{SQS}(n)$}

In this section, we describe the classical doubling construction of $\mathrm{SQS}(2 n)$ from given SQS $(n)$. Both constructions were described in [8], which we give here almost without changes. Denote by $F=F_{1}, F_{2}, \ldots, F_{n-1}$ a full partition of $E_{2}^{n}$ into subcodes with distance 4 , i.e. for any $i, i=1, \ldots, n-1$ the set $F_{i}$ is a constant weight $(n, 2,4, n / 2)$-code. Let $F$ and $H$ be any such partitions of $E_{2}^{n}$, where $H=H_{1}, \ldots, H_{n-1}$.

Construction $A^{*}$. Let $(X, A)$ and $(Y, B)$ be any two Steiner systems $S(n)=S(n, 4,3)$ where $X \cap Y=\emptyset$. Let $F$ and $H$ where $F=F_{1}, \ldots, F_{n-1}$ and $H=H_{1}, \ldots, H_{n-1}$ be any full partitions of $E_{2}^{n}$ and let $\alpha$ be any permutation from $S_{n}$. Define a constant weight code $S$ on coordinate set $Q=X \cup Y$ as follows:

(1) Any codeword belonging to $A$ or $B$ belongs to $S$;

(2) if $i_{1}, i_{2} \in X$ and $j_{1}, j_{2} \in Y$ then $\boldsymbol{c}$ with $\operatorname{supp}(\boldsymbol{c})=\left\{i_{1}, i_{2}, j_{1}, j_{2}\right\}$ is a codeword of $C$, if and only if $\in \mathbf{F}_{\mathbf{i}}$ with $\operatorname{supp}(\boldsymbol{f})=\left\{i_{1}, i_{2}\right\}, \boldsymbol{h} \in H_{j}$ with $\operatorname{supp}(\boldsymbol{h})=\left\{j_{1}, j_{2}\right\}$ and $\alpha(i)=j$.

Proposition 1 [8] Under construction, described above the set $(Q, S)$ is a Steiner system $S(2 n, 4,3)$.

In [7] these authors, using this construction and knowledge of all automorphisms groups of these partitions, derived the lower bound for $N(16) \geq 31021$.

Denote by $F_{1}, F_{2}, \ldots, F_{6}$ the all non-isomorphic 1-partitions of $E_{2}^{8}$, obtained in [22,23]. Agree that $F_{5}$ and $F_{6}$ are two partitions, not containing a sub-partitions of index 2 (see [24]), i.e. subcodes of partitions $F_{5}$ (respectively, $F_{6}$ ) do not form a partition of $E_{2}^{4}$ for any choice of 4 positions from $E_{2}^{8}$.

Denote by $\left\{F_{i}\right\}$ the orbit by action of $S_{8}$ on $F_{i}$ :

$$
\left\{F_{i}\right\}=\operatorname{Orb}_{S_{8}}\left(F_{i}\right), \quad i=1, \ldots, 6 .
$$

Simple arguments show that [7] for any two fixed $S(8,4,3)$ systems $(X, A)$ and $(Y, B)$ there are at least $\left|\left\{F_{i}\right\}\right| \cdot\left|\left\{F_{j}\right\}\right| \cdot 7$ ! distinct $S(16,4,3)$ obtained by Construction $A^{*}$ by taking any $F_{i} \in\left\{F_{i}\right\}$ for $F$ and any $F_{j} \in\left\{F_{j}\right\}$ for $G$ with $j \in\{5,6\}$ and $i \neq j$. In addition, every such $S(16,4,3)$ has exactly two subsystems $S(8,4,3)$, namely $(X, A)$ and $(Y, B)$. It follows [7] that there are at least

$$
N_{A^{*}}=\frac{7 !}{1344^{2}}\left(\sum_{i=1}^{5}\left|\left\{F_{i}\right\}\right| \cdot\left|\left\{F_{6}\right\}\right|+\sum_{i=1}^{4}\left|\left\{F_{i}\right\}\right| \cdot\left|\left\{F_{5}\right\}\right|\right) .
$$


By this construction it can be seen [7] that all resulting systems $S(16,4,3)$ have rank exacxtly 14 over $\mathbb{F}_{2}$. The exact computation shows [7] that $N_{A^{*}}=31021$. Using 280 systems found in [25], for which the number of sub-systems $S(8,4,3)$ different from two (which means that these 280 systems $S(16,4,3)$ have the rank less or equal to 13 over $\left.\mathbb{F}_{2}\right)$, one can get [7] that $N(16) \geq 31301$.

\section{$\S 4$. General properties of SQS(16) with rank 14 over $\mathbb{F}_{2}$}

Let $S$ be an arbitrary Steiner system $S(16,4,3)$ of rank 14 over $\mathbb{F}_{2}$. We consider the general properties of such system.

Applying the appropriate permutation of coordinates, $S$ can be presented in the form, when the $[16,8,2]$-code $S^{\perp}$, dual to $S$, looks as follows:

$$
S^{\perp}=\left\{\boldsymbol{u}_{0}, \boldsymbol{u}_{1}, \boldsymbol{u}_{2}, \boldsymbol{u}_{1}+\boldsymbol{u}_{2}\right\}
$$

where $\boldsymbol{u}_{0}$ is the zero vector, $\boldsymbol{u}_{1}=(1111111100000000)$, and $\boldsymbol{u}_{2}=(0000000011111111)$. Thus we split coordinates of $S$ into two blocks of eight coordinates such that any $\boldsymbol{c} \in S$ consists of two vectors $\boldsymbol{c}=\left(\boldsymbol{c}_{1} \mid \boldsymbol{c}_{2}\right)$ where each vector $\boldsymbol{c}_{i}$ satisfies to the overall parity checking:

$$
\mathrm{wt}\left(\boldsymbol{c}_{i}\right) \equiv 0(\bmod 2), \quad i=1,2
$$

(we call it a parity rule).

Definition 2 Let $S$ be a Steiner system $(16,4,3)$ of rank 14 over $\mathbb{F}_{2}$ with dual code (2). Define the subset $S_{u v}$ of $S$ where $u, v \in\{0,2,4\}$ as follows:

$$
S_{u v}=\{\boldsymbol{c}=(\boldsymbol{a} \mid \boldsymbol{b}) \in S: \operatorname{wt}(\boldsymbol{a})=u, \operatorname{wt}(\boldsymbol{b})=v\} .
$$

These words are called $(u, v)$-words.

Lemma 1 Let $S$ be a Steiner system $(16,4,3)$ of rank 14 over $\mathbb{F}_{2}$ with dual code (2). Then $S$ is a union of three subsets

$$
S=S_{40} \bigcup S_{04} \bigcup S_{22}
$$

where $S_{40}$ (respectively $\left.S_{04}\right)$ is a Steiner system $S(8,4,3)$ and $S_{22}$ has cardinality 112 .

Proof. Follows from definition of Steiner system $S(16,4,3)$.

The group (subgroup of $S_{16}$ ) of two elements which permutes the blocks is identified with $S_{2}$. An element $\tau_{1} \times \tau_{2} \in S_{8} \times S_{8} \subset S_{16}$ acts on $(\boldsymbol{x} \mid \boldsymbol{y})$ in the natural way:

$$
\left(\tau_{1} \times \tau_{2}\right)(\boldsymbol{x} \mid \boldsymbol{y})=\left(\tau_{1}(\boldsymbol{x}) \mid \tau_{2}(\boldsymbol{y})\right) .
$$

We have the following statement. 
Lemma 2 Let $S$ be an arbitrary Steiner system $S(16,4,3)$ of rank 14 over $\mathbb{F}_{2}$ with dual code (2). Suppose there exists a permutation $\sigma \in S_{16}$ so that $\sigma S$ satisfies the parity rule. Then $\sigma \in S_{2} \rtimes\left(S_{8} \times S_{8}\right)$.

Proof. Since $S$ satisfies parity rule, we have that

$$
\left(\boldsymbol{x} \cdot \boldsymbol{u}_{1}\right)=0
$$

for any $\boldsymbol{x} \in S$. Similarly, since $\sigma S$ satisfies the parity rule, we have that

$$
\left(\sigma(\boldsymbol{x}) \cdot \boldsymbol{u}_{1}\right)=0, \quad \text { for any } \boldsymbol{x} \in S .
$$

Multiplying both vectors $\sigma(\boldsymbol{x})$ and $\boldsymbol{u}_{1}$ by $\sigma^{-1}$, we obtain

$$
\left(\boldsymbol{x} \cdot \sigma^{-1}\left(\boldsymbol{u}_{1}\right)\right)=0, \quad \text { for any } \boldsymbol{x} \in S .
$$

Let $\boldsymbol{u}^{\prime}=\boldsymbol{u}_{1}+\sigma^{-1}\left(\boldsymbol{u}_{1}\right)$. From (3) and (4) we have that

$$
\left(\boldsymbol{x} \cdot \boldsymbol{u}^{\prime}\right)=0, \quad \text { for any } \boldsymbol{x} \in S .
$$

Thus $\boldsymbol{u}^{\prime} \in S^{\perp}$ and consequently (recall that $S^{\perp}$ is a vector space) $\sigma^{-1}\left(\boldsymbol{u}_{1}\right) \in S^{\perp}$. Taking into account that $\sigma^{-1}\left(\boldsymbol{u}_{1}\right)$ is of weight 8 , we obtain that $\sigma^{-1}\left(\boldsymbol{u}_{1}\right)$ is equal to either $\boldsymbol{u}_{1}$ or $\boldsymbol{u}_{2}$. So $\sigma\left(\boldsymbol{u}_{1}\right)=\boldsymbol{u}_{1}$ or $\sigma\left(\boldsymbol{u}_{2}\right)=\boldsymbol{u}_{1}$, in other words, $\sigma$ either stabilizes the blocks or permutes them.

Recall that $E_{2}^{8}$ is the subset of $E^{8}$, formed by the all vectors of weight 2. Denote any codeword of $S$ by $\boldsymbol{c}=(\boldsymbol{a} \mid \boldsymbol{b})$.

Definition 3 Let $S$ be a Steiner system $(16,4,3)$ of rank 14 over $\mathbb{F}_{2}$ with dual code (2). Let $\boldsymbol{c}=(\boldsymbol{a} \mid \boldsymbol{b})$ be any codeword of $S$ such that $\mathrm{wt}(\boldsymbol{a})=\operatorname{wt}(\boldsymbol{b})$. Denote by $A_{l}(\boldsymbol{b})$ (respectively, by $A_{r}(\boldsymbol{a})$ ) the sets obtained by fixing vector $\boldsymbol{b}$ (respectively $\left.\boldsymbol{a}\right)$ :

$$
A_{r}(\boldsymbol{a})=\{\boldsymbol{b}:(\boldsymbol{a} \mid \boldsymbol{b}) \in S\}, \quad A_{l}(\boldsymbol{b})=\{\boldsymbol{a}:(\boldsymbol{a} \mid \boldsymbol{b}) \in S\} .
$$

Lemma 3 Suppose the conditions of lemma 2 are satisfied. Let $\boldsymbol{c}=(\boldsymbol{a} \mid \boldsymbol{b})$ be any codeword of $S$ such that $\operatorname{wt}(\boldsymbol{a})=\operatorname{wt}(\boldsymbol{b})$. Then the set $A_{l}(\boldsymbol{b})$ (respectively $A_{r}(\boldsymbol{a})$ ) is a Steiner system $S(8,2,1)$ (or, equivalently, a constant weight $(8,2,4,4)$ code).

Proof. The fact that $A_{l}(\boldsymbol{b})$ (respectively, $A_{r}(\boldsymbol{a})$ ) is a constant weight code $\left(8,2,4, N_{l}(\boldsymbol{b})\right.$ ) with minimal distance 4 follows from definition of such set. Indeed, any two words of $S$ have distance not less than 4, implying that any two distinct words $\boldsymbol{x}$ and $\boldsymbol{x}^{\prime}$ of $A_{l}(\boldsymbol{b})$ have distance not less than 4. From the other side, since $S$ is a 3-design, nonzero positions of vectors $\boldsymbol{x}$ from $A_{l}(\boldsymbol{b})$ should cover all 8 positions of the first coordinate block of $S$. This means that for any $\boldsymbol{b} \in E_{2}^{8}$ the set $A_{l}(\boldsymbol{b})$ is a 1-design or $S(8,2,1)$. This follows also from counting arguments. In average, over all $\boldsymbol{b} \in E_{2}^{8}$, we have that

$$
\left|\bar{A}_{l}\right|=\frac{1}{\left|E_{2}^{8}\right|} \times \sum_{\boldsymbol{b} \in E_{2}^{8}}\left|A_{l}(\boldsymbol{b})\right|=\frac{\left|C_{(2)}\right|}{\left|E_{2}^{8}\right|}=4 .
$$

From the other side, $\left|A_{l}(\boldsymbol{b})\right|$ can not be more than 4 for any $\boldsymbol{b} \in E_{2}^{8}$. Thus $\left|A_{l}(\boldsymbol{b})\right|=4$. Similarly, the same equality is valid for $\left|A_{r}(\boldsymbol{a})\right|$. 
Definition 4 Define the sphere $W_{i} \subset E_{2}^{8}, i=1,2, \ldots, 8$ of radius two as a set of seven vectors $\boldsymbol{e}_{1}(i), \ldots, \boldsymbol{e}_{7}(i)$ from $E_{2}^{8}$, which satisfy to the following properties:

1). $\{i\} \in \operatorname{supp}\left(\boldsymbol{e}_{j}(i), j=1, \ldots 7\right.$.

2). $d\left(\boldsymbol{e}_{j}(i), \boldsymbol{e}_{s}(i)\right)=2$, for any $j \neq s$.

For example, the sphere $W_{8}$, which we use very often, consists of the following vectors, which we denote for short $\boldsymbol{e}_{s}(8)=\boldsymbol{e}_{s}$ :

$$
\begin{aligned}
& \boldsymbol{e}_{1}=(00000011), \quad \boldsymbol{e}_{2}=(00000101), \\
& \boldsymbol{e}_{3}=(00001001), \quad \boldsymbol{e}_{4}=(00010001), \\
& \boldsymbol{e}_{5}=(00100001), \quad \boldsymbol{e}_{6}=(01000001), \\
& \boldsymbol{e}_{7}=(10000001) .
\end{aligned}
$$

Note that the stabilizer of $W_{8}$ in $S_{8}$ fixes the last nonzero coordinate of $\boldsymbol{e}_{i}(8)$ and is isomorphic to $S_{7}$.

Lemma 4 Suppose we are in conditions of lemma 2 and let $\left(\boldsymbol{a}_{1} \mid \boldsymbol{b}_{1}\right)$ and $\left(\boldsymbol{a}_{2} \mid \boldsymbol{b}_{2}\right)$ be any two codewords of $C_{(2)}$. Let $\boldsymbol{a}_{1}$ and $\boldsymbol{a}_{2}$ (respectively, $\boldsymbol{b}_{1}$ and $\boldsymbol{b}_{2}$ ) be such that $d\left(\boldsymbol{a}_{1}, \boldsymbol{a}_{2}\right)=2$ (respectively, $d\left(\boldsymbol{b}_{1}, \boldsymbol{b}_{2}\right)=2$ ). Then the corresponding codes $A_{r}\left(\boldsymbol{a}_{1}\right)$ and $A_{r}\left(\boldsymbol{a}_{2}\right)$ (respectively, $A_{l}\left(\boldsymbol{b}_{1}\right)$ and $\left.A_{l}\left(\boldsymbol{b}_{2}\right)\right)$ do not intersect each other, i.e. $A_{r}\left(\boldsymbol{a}_{1}\right) \cap A_{r}\left(\boldsymbol{a}_{2}\right)=\emptyset$ (respectively, $\left.A_{l}\left(\boldsymbol{b}_{1}\right) \cap A_{l}\left(\boldsymbol{b}_{2}\right)=\emptyset\right)$.

Proof. In contrary, assume that there is $\boldsymbol{x}$ such that $\boldsymbol{x} \in A_{r}\left(\boldsymbol{a}_{1}\right) \cap A_{r}\left(\boldsymbol{a}_{2}\right)$. Then we have

$$
d\left(\left(\boldsymbol{a}_{1} \mid \boldsymbol{x}\right),\left(\boldsymbol{a}_{2} \mid \boldsymbol{x}\right)\right)=d\left(\boldsymbol{a}_{1}, \boldsymbol{a}_{2}\right)=2,
$$

i.e. a contradiction, since $\left(\boldsymbol{a}_{1} \mid \boldsymbol{x}\right)$ and $\left(\boldsymbol{a}_{2} \mid \boldsymbol{x}\right)$ are distinct codewords of $C$. The proof of the second statement is similar.

Lemma 5 Suppose we are in conditions of lemma 2 and let $W_{i}=\left\{\boldsymbol{e}_{1}(i), \ldots, \boldsymbol{e}_{7}(i)\right\}$ be any sphere, $i=1,2, \ldots, 8$. Then the set of codes $A_{l}\left(\boldsymbol{e}_{1}(i)\right), A_{l}\left(\boldsymbol{e}_{2}(i)\right), \ldots, A_{l}\left(\boldsymbol{e}_{7}(i)\right)$ forms a partition of $E_{2}^{8}$.

Proof. Since

$$
\left|W_{i}\right| \times\left|A_{l}\left(\boldsymbol{e}_{s}(i)\right)\right|=\left|E_{2}^{8}\right|=28,
$$

we have to check only that any two distinct codes $A_{l}\left(\boldsymbol{e}_{j}(i)\right)$ and $A_{l}\left(\boldsymbol{e}_{s}(i)\right)$ where $j \neq s$ and $j, s \in\{1, \ldots, 7\}$ have empty intersection. But this follows from lemma 4 , since for any $\boldsymbol{e}_{j}(i), \boldsymbol{e}_{s}(i)$ from $W_{i}$ we have that $d\left(\boldsymbol{e}_{j}(i), \boldsymbol{e}_{s}(i)\right)=2$.

Remark 1 It is easy to see that the results above, which we derived for Steiner system $S(16,4,3)$ of rank 14 over $\mathbb{F}_{2}$, are valid for any $S(n, 4,3)$ of arbitrary order $n \geq 16$ with rank $n-2$ over $\mathbb{F}_{2}$ such that $n / 2 \equiv 2$ or $4(\bmod 6)$.

\section{$\S 5$. Generalized doubling construction of $S(16,4,3)$ with rank 14 over $\mathbb{F}_{2}$}


Now we describe the general doubling construction of Steiner systems $S(16,4,3)$ with rank 14 over $\mathbb{F}_{2}$. This construction is induced by the general doubling construction of the extended binary perfect nonlinear $\left(16,4,2^{11}\right)$-codes of rank 14 over $\mathbb{F}_{2}$, which we described in [15]. Indeed, the set of codewords of weight four of any such $\left(16,4,2^{11}\right)$-code with zero codeword forms a Steiner system $S(16,4,3)$.

It is convenient for us to present such a system $S(16,4,3)$ by the corresponding constant weight $(16,4,4,140)$ code, which uniquely defines this system [16], and which we denote here by $S$. Denote by $\mathcal{S}$ the set of all such distinct $(16,4,4,140)$ codes $S$. Our purpose now is to parameterize all these Steiner systems, using the canonical partitions of $E_{2}^{8}$. We can do it using the special subsets of $S$, called headings, formed by the two partitions, connected with the two spheres $W_{8}=\left\{\boldsymbol{e}_{s}: s=1, \ldots, 7\right\}$ which occur on the left and right hand sides (the first and the second blocks) of the codewords. We start with the definition of heading of a code. Clearly when $\boldsymbol{c}=(\boldsymbol{a} \mid \boldsymbol{b})$ runs over $S$, each of two vectors $\boldsymbol{a}$ and $\boldsymbol{b}$ run over the set $E_{2}^{8}$. In particular, when $\boldsymbol{a}$ runs over the sphere $W_{8}$ the corresponding codes $A_{r}(\boldsymbol{a})$ form a partition of $E_{2}^{8}$,

$$
E_{2}^{8}=\bigcup_{\boldsymbol{a} \in W_{8}} A_{r}(\boldsymbol{a})=\bigcup_{s=1}^{7} A_{r}\left(\boldsymbol{e}_{s}\right) .
$$

Similarly, when $\boldsymbol{b}$ runs over the set $W_{8}$, the codes $A_{l}(\boldsymbol{b})$ also form a partition of $E_{2}^{8}$.

Denote by $\Omega$ the set of all distinct partitions $L_{i}=\left(L_{i, 1}, L_{i, 2}, L_{i, 3}, L_{i, 4}\right)$ of $E_{2}^{8}$ into (binary constant weight) $(8,2,4,4)$ codes $L_{i, s}, s=1,2,3,4$. Moreover the following result holds.

Proposition 2 (Computational result). There exist exactly 6240 different partitions of $E_{2}^{8}$ which can be arranged under action of $S_{8}$ into six orbits $\operatorname{Orb}_{S_{8}}\left(L_{i}\right)$, ordered according to the indices $i$ of $\mathrm{Orb}_{S_{8}}\left(L_{i}\right)$.

We assume that the unique Steiner system $S(8,4,3)$ is formed by the following vectors (in addition to words of all zeroes and ones):

$$
\begin{array}{ll}
(1111 \mid 0000), & (0000 \mid 1111), \\
(1100 \mid 1100), & (0011 \mid 0011), \\
(1100 \mid 0011), & (0011 \mid 1100), \\
(1010 \mid 0110), & (0101 \mid 1001), \\
(1010 \mid 1001), & (0101 \mid 0110), \\
(1001 \mid 1010), & (0110 \mid 0101), \\
(1001 \mid 0101), & (0110 \mid 1010) .
\end{array}
$$

Denote by $P$ its stabilizer in $S_{8}$ and by $P^{\prime}$ its stabilizer in the group $S_{7}$.

Definition 5 Define the group:

$$
G=S_{2} \rtimes(P \times P) \subset S_{16}
$$


It is known (see, for example, [8]) that $|P|=1344$. Recall Lemma 1 that any system $S$ of rank 14 (with dual code $S^{\perp}$ given by (2)) is partitioned into three subsets $S_{40}, S_{04}$ and $S_{22}$. Without loss of generality, we can assume from now that all our systems $S$ from $\mathcal{S}$ are such that the subsets $S_{40}=A_{l}(\mathbf{0})$ and $S_{04}=A_{r}(\mathbf{0})$ of 14 elements are obtained from the Steiner system given above. This condition increases the number of non-equivalent partitions of $E_{2}^{8}$ since we consider the $P$-equivalence and $P^{\prime}$-equivalence.

Proposition 3 (Computational result). Let $\Omega$ be the set of all 6240 different partitions of $E_{2}^{8}$ into $(8,2,4,4)$ codes. Then $\Omega$ splits into $43 P$-orbits $\operatorname{Orb}_{P}\left(L_{i}\right)(i=1, \ldots, 43)$ and 62 $P^{\prime}$-orbits $\operatorname{Orb}_{P^{\prime}}\left(L_{i}^{\prime}\right)$. We assume that the 62 non-equivalent partitions $L_{i}^{\prime}$ are chosen so that $L_{i}=L_{i}^{\prime}$, where $i=1, \ldots, 43$.

We denote $L_{i}^{\prime}$ via $L_{i}, i=1, \ldots, 62$ and call them canonical partitions of $E_{2}^{8}$.

For any such canonical partition $L_{i}$, denote by $\operatorname{Stab}_{P}\left(L_{i}\right)$ the stabilizer of $L_{i}$ in the group $P$ and by $Q_{i} \subset S_{7}$ a group of permutations of its seven components $L_{i, 1}, L_{i, 2}, \ldots, L_{i, 7}$ induced by the automorphisms of $P$ :

$$
Q_{i}=\left\{\pi \in S_{7}: \exists \boldsymbol{g} \in \operatorname{Stab}_{P}\left(L_{i}\right): \boldsymbol{g} L_{i, s}=L_{i, \pi^{-1}(s)}, i=1, \ldots, 7\right\} .
$$

For an element $\boldsymbol{a} \in E^{8}$ and set $X \subseteq E^{8}$ denote:

$$
\boldsymbol{a} \times X=\{(\boldsymbol{a} \mid \boldsymbol{x}): \boldsymbol{x} \in X\}, \quad X \times \boldsymbol{a}=\{(\boldsymbol{x} \mid \boldsymbol{a}): \boldsymbol{x} \in X\}
$$

Definition 6 Let $S$ be a $(16,4,4,140)$ code with rank 14 over $\mathbb{F}_{2}$. Define the following subset $F=F(S)$ of $S$ (of 56 words), consisting of two partitions (with 7 common words counted twice)

$$
F(S)=\bigcup_{s=1}^{7}\left\{\left(\boldsymbol{e}_{s} \mid \boldsymbol{y}\right): \boldsymbol{y} \in A_{r}\left(\boldsymbol{e}_{s}\right)\right\} \bigcup \bigcup_{s=1}^{7}\left\{\left(\boldsymbol{x} \mid \boldsymbol{e}_{s}\right): \boldsymbol{x} \in A_{l}\left(\boldsymbol{e}_{s}\right)\right\}
$$

We say that $S$ has a heading $F$ and for the sake of simplicity write as:

$$
F=\bigcup_{s=1}^{7} \boldsymbol{e}_{s} \times A_{r}\left(\boldsymbol{e}_{s}\right) \bigcup \bigcup_{s=1}^{7} A_{l}\left(\boldsymbol{e}_{s}\right) \times \boldsymbol{e}_{s}
$$

Assume that the partition $A_{l}\left(\boldsymbol{e}_{1}\right), \ldots, A_{l}\left(\boldsymbol{e}_{7}\right)$ is equivalent to $L_{i}$ for some $i, i=1, \ldots, 43$ and the partition $A_{r}\left(\boldsymbol{e}_{1}\right), \ldots, A_{r}\left(\boldsymbol{e}_{7}\right)$ is equivalent to $L_{j}$ for some $j, j=1, \ldots, 62$. Recall that $L_{i}$ (respectively, $L_{j}$ ) are among of the 43 (respectively 62) canonical (non-equivalent) partitions, given by proposition 3 . All these partitions $L_{i}, i=1, \ldots, 62$ are ordered, according to the vectors $\boldsymbol{e}_{s}$ of the ball $W_{8}$ :

$$
L_{i}=\left(L_{i, 1}, \ldots, L_{i, 7}\right) \text { where } \boldsymbol{e}_{s} \in L_{i, s} \text { for } s=1, \ldots, 7
$$


Without loss of generality we can assume that $i \leq j$ (if not we can consider the Steiner system $S^{\prime}$ obtained from $S$ by switching the sides). Furthermore, by the corresponding permutation of coordinates we can obtain the following ordering of $L_{i}$ :

$$
L_{i}=\left(L_{i, 1}, \ldots, L_{i, 7}\right), \quad L_{i, s}=A_{l}\left(\boldsymbol{e}_{s}\right),
$$

where the vectors $\boldsymbol{e}_{s}(s=1, \ldots, 7)$ are given by definition 4 . In such way we arrive to the following natural canonical heading.

Definition 7 (Canonical $(i, j, k)$ heading). Let $1 \leq i \leq 43$ and $i \leq j \leq 62$ and $L_{i}, L_{j}$ are two canonical partitions. Define the set of 56 (where 7 words are counted twice) elements as follows:

$$
\begin{aligned}
F_{i, j}^{(k)} & =\bigcup_{s=1}^{7} L_{i, \pi(s)} \times \boldsymbol{e}_{s} \bigcup \bigcup_{s=1}^{7} \boldsymbol{e}_{\pi(s)} \times L_{j, s} \\
& =\bigcup_{s=1}^{7}\left\{\left(\boldsymbol{x} \mid \boldsymbol{e}_{s}\right): \boldsymbol{x} \in L_{i, \pi(s)}\right\} \bigcup_{s=1}^{7}\left\{\left(\boldsymbol{e}_{\pi(s)} \mid \boldsymbol{y}\right): \boldsymbol{y} \in L_{j, s}\right\} .
\end{aligned}
$$

where $\pi=\pi_{k}^{-1}, k=1,2, \ldots, m(i, j)$, and

$$
\left\{\pi_{1},, \pi_{2}, \ldots, \pi_{m(i, j)}\right\}
$$

is a fixed set of the $\left(Q_{j}-Q_{i}\right)$-double-coset representatives of $S_{8}$.

We know all canonical headings $(i, j, k)$.

Proposition 4 (Computational result). There exist 339716 different canonical headings $(i, j, k)$.

Using canonical headings, now we can define canonical Steiner systems $C$.

Definition 8 (Canonical Steiner system). Let $S$ be any Steiner system from $\mathcal{S}$. We say that $S$ is a canonical $(i, j, k)$ code, denoted by $S_{i, j}^{(k)}$ if $S$ has a canonical heading

$$
F\left(S_{i, j}^{(k)}\right)=F_{i, j}^{(k)}
$$

Now the important question is does any system $S$ from $\mathcal{S}$ equivalent to a canonical one $S_{i, j}^{(k)} ?$

Proposition 5 Let $S \in \mathcal{S}$ and let $F=F(S)$ be the heading of $S$. Then $S$ is $G$-equivalent to a canonical Steiner system $S_{i, j}^{(k)} \in \mathcal{S}$ with heading $F_{i, j}^{(k)}$, where $1 \leq i \leq 43$ and $i \leq j \leq 62$ and where the permutation $\pi_{k}$ is defined by definition 7. 
Proof. Let $S$ be any Steiner system from $\mathcal{S}$. Define the following subset of $S$

$$
Y_{2}=\bigcup_{s=1}^{7} A_{l}\left(\boldsymbol{e}_{s}\right) \times \boldsymbol{e}_{s}=\left\{\left(\boldsymbol{y} \mid \boldsymbol{e}_{s}\right): \boldsymbol{e}_{s} \in W_{8}, \boldsymbol{y} \in A_{l}\left(\boldsymbol{e}_{s}\right)\right\},
$$

where $A_{l}\left(\boldsymbol{e}_{s}\right), s=1, \ldots, 7$ is a partition $A_{l}$ of $E_{2}^{8}$. Assume that $A_{l}$ is $P$-equivalent to $L_{i}$ for some $i$. Thus there exists a permutation $\tau_{2} \in P$ such that $\tau_{2} A_{l}=L_{i}$ and in particular

$$
\tau_{2} A_{l}\left(\boldsymbol{e}_{s}\right)=L_{i, \tau_{2}^{-1}(s)}
$$

Let $1_{8}$ be the identity element of $S_{8}$. Applying the element $\tau_{2} \times 1_{8}$ to $S$, its subset defined (7), and taking into account (8), we have

$$
\begin{aligned}
\left(\tau_{2} \times 1_{8}\right) Y_{2} & =\left(\tau_{2} \times 1_{8}\right)\left\{\bigcup_{s=1}^{7} A_{l}\left(\boldsymbol{e}_{s}\right) \times \boldsymbol{e}_{s}\right\} \\
& =\bigcup_{s=1}^{7}\left(\tau_{2} A_{l}\left(\boldsymbol{e}_{s}\right)\right) \times \boldsymbol{e}_{s} \\
& =\bigcup_{s=1}^{7} L_{i, \tau_{2}^{-1}(s)} \times \boldsymbol{e}_{s} \\
& =\bigcup_{s=1}^{7} L_{i, s} \times \boldsymbol{e}_{\tau_{2}(s)} .
\end{aligned}
$$

Set $S^{\prime}=\left(\tau_{2} \times 1_{8}\right) S$, and define the following subset of $S^{\prime}$

$$
Y_{1}=\bigcup_{s=1}^{7} \boldsymbol{e}_{s} \times A_{r}\left(\boldsymbol{e}_{s}\right)=\left\{\left(\boldsymbol{e}_{s} \mid \boldsymbol{y}\right): \boldsymbol{e}_{s} \in W_{8}, \boldsymbol{y} \in A_{r}\left(\boldsymbol{e}_{s}\right)\right\}
$$

where $A_{r}\left(\boldsymbol{e}_{s}\right), s=1, \ldots, 7$ is a partition $A_{r}$ of $E_{2}^{8}$. Assume that $A_{r}$ is $P^{\prime}$-equivalent to $L_{j}$ for some $j$. Thus there exists an element $\tau_{1} \in P^{\prime}$ such that $\tau_{1} A_{r}=L_{j}$ and $\tau_{1} W_{8}=W_{8}$. In particular

$$
\tau_{1} A_{r}\left(\boldsymbol{e}_{s}\right)=L_{j, \tau_{1}^{-1}(s)} .
$$

Applying the element $1_{8} \times \tau_{1}$ to $S^{\prime}$, its subset $Y_{1}$, and taking into account (9), we have

$$
\begin{aligned}
\left(1_{8} \times \tau_{1}\right) Y_{1} & =\left(1_{8} \times \tau_{1}\right)\left\{\bigcup_{s=1}^{7} \boldsymbol{e}_{s} \times A_{r}\left(\boldsymbol{e}_{s}\right)\right\} \\
& =\bigcup_{s=1}^{7} \boldsymbol{e}_{s} \times\left(\tau_{1} A_{r}\left(\boldsymbol{e}_{s}\right)\right) \\
& =\bigcup_{s=1}^{7} \boldsymbol{e}_{s} \times L_{j, \tau_{1}^{-1}(s)} .
\end{aligned}
$$


Moreover, we have

$$
\begin{aligned}
\left(1_{8} \times \tau_{1}\right)\left\{\bigcup_{s=1}^{7} L_{i, s} \times \boldsymbol{e}_{\tau_{2}(s)}\right\} & =\bigcup_{s=1}^{7} L_{i, s} \times \tau_{1}\left(\boldsymbol{e}_{\tau_{2}(s)}\right) \\
& =\bigcup_{s=1}^{7} L_{i, s} \times \boldsymbol{e}_{\tau_{3}(s)}
\end{aligned}
$$

for some permutation $\tau_{3} \in S_{7}$. Since $\boldsymbol{e}_{\tau_{3}(s)} \in L_{j, \tau_{1}^{-1}(s)}$ we conclude that $\tau_{3}=\tau_{1}^{-1}$. Set $S^{\prime \prime}=\left(1_{8} \times \tau_{1}\right) S^{\prime}$. Then $S^{\prime \prime}$ is equivalent to $S$ and its heading by definition is equal to

$$
\bigcup_{s=1}^{7} L_{i, s} \times \boldsymbol{e}_{\tau_{1}^{-1}(s)} \bigcup \bigcup_{s=1}^{7} \boldsymbol{e}_{s} \times L_{j, \tau_{1}^{-1}(s)} .
$$

Without loss of generality we can always assume that $i \leq j$ (if not apply the permutation of $S_{2}$ from the definition of $G$, i.e. switch the blocks of coordinates).

It is clear that a Steiner system $S$ can have different headings as well as different Steiner systems may have the same heading.

Now we want to describe the general doubling construction of Steiner systems $S(16,4,3)$ of rank 14 over $\mathbb{F}_{2}$.

Definition 9 Let $\mathcal{M}_{s}, s=1,2, \ldots, 7$ be the set of constant weight $(8,2,4,4)$ codes containing $e_{s}$. Let

$$
\mathcal{M}=\bigcup_{s=1}^{7} \mathcal{M}_{s}
$$

be the set of all constant weight $(8,2,4,4)$ codes.

It is easy to check that there are 15 codes in every set $\mathcal{M}_{s}$ so that the total number of $(8,2,4,4)$ codes is 105 . We consider functions from $E_{2}^{8}$ to $\mathcal{M}$.

Definition 10 (Admissible function) We say that a function $\Lambda: E_{2}^{8} \rightarrow \mathcal{M}$ is admissible if there exist $1 \leq i \leq 43, i \leq j \leq 62$, and a permutation $\pi_{k}$ such that:

1). $\Lambda\left(\boldsymbol{e}_{\pi^{-1}(s)}\right)=L_{j, s}$, for $s=1, \ldots, 7$.

2). $\Lambda(\boldsymbol{x})=M \in \mathcal{M}_{s}$, where $\boldsymbol{x} \in L_{i, \pi^{-1}(s)}$ and $s=1, \ldots, 7$.

Such function will be called an $(i, j, k)$-admissible function.

Admissible functions are used to parameterize canonical Steiner systems. Indeed for any canonical Steiner system $S=S_{i, j}^{(k)}$, and any $\boldsymbol{x} \in E_{2}^{8}$, set $\Lambda(\boldsymbol{x})=A_{r}(\boldsymbol{x})$ (see Definition 3 ). Then

$$
S_{22}=\bigcup_{\boldsymbol{x} \in E_{2}^{8}} \boldsymbol{x} \times \Lambda(\boldsymbol{x}),
$$

where $\Lambda$ is $(i, j, k)$-compatible by definition. 


\section{$\S 6 . \quad$ Derived triple systems}

For an $\operatorname{SQS}(v)$, given by the pair of sets $(X, B)$, a derived triple system (briefly DTS $(v-$ $1)$ ) of $(X, B)$ is a pair $\left(X_{a}, B_{a}\right)$, where $X_{a}=X \backslash\{a\}$ and $B_{a}=\{b \backslash\{a\}: a \in b \in B\}$. It is obvious, that every derived triple system is a Steiner triple system $S(v-1,3,2)$. For $v=16$ we obtain a system $S(15,3,2)$. It is known [19] from 1917 that there are exactly 80 non-isomorphic systems $S(15,3,2)$. There is a standard numbering of these systems by the indices from 1 to 80, related to the number of Pasch configurations (see [1]).

Given a Steiner system $S=S(v, 4,3)$, let $\beta=\beta(S)$ denote the number of its pairwise non-isomorphic DTS $(v-1)$. Clearly $1 \leq \beta \leq v$ for any $\operatorname{SQS}(v)$. A system $\operatorname{SQS}(v)$ is said to be homogeneous (respectively, heterogeneous), if $\beta=1$ (respectively, $\beta=v$ ). Among all Steiner systems SQS(16) of rank at most thirteen, the only derived systems DTS(15) that we found are those with numbers $1,2,3,4,5,6,7$. All Steiner triple systems with these numbers occur as the DTS(15) in the homogeneous SQS(16).

Denote by $N_{\text {hom }}(i)$ the number of non-isomorphic homogeneous systems SQS(16) with rank at most thirteen, whose derived systems are DTS(15) with number $i$, where $i \in$ $\{1,2, \ldots, 7\}$. Denote by $N(\beta)$ the number of such non-isomorphic systems SQS(16) with rank at most thirteen with given $\beta$. Denote by $N\left(\mu\left(i_{1}\right), \mu\left(i_{2}\right), \ldots, \mu\left(i_{\beta}\right)\right)$ the number of non-isomorphic systems SQS(16) with rank at most thirteen which have $\mu\left(i_{s}\right)>0$ derived systems with number $i_{s}$, where $i_{s} \in\{1,2, \ldots, 7\}$ for $s=1, \ldots, \beta$, i.e. in our notation $N_{\text {hom }}(i)=N(\mu(i)=16)$.

\section{$\S 7$. Non-isomorphic Steiner systems SQS(16) of rank 14 over $\mathbb{F}_{2}$}

Theorem 1 There exists 684764 non-equivalent Steiner systems S(16,4,3) of length 16 and rank 14 .

Proof. Computational result. First, we construct all different Steiner systems using $(i, j, k)$-admissible functions $\Lambda$. Then to any Steiner system SQS(16) we associate a set of 16 indices of the derived triple systems. We note that if the two sets that correspond to an arbitrary two SQS(16) systems are different these systems are non-equivalent. Thus all different Steiner systems are arranged into lists which correspond to the same set of 16 indices. The lists are pair-wise non-equivalent, i.e. two systems belong to the different lists are non-equivalent.

\section{$\S 8$. Resolvability}

The general resolvability problem for $\operatorname{SQS}(v)$ can be stated as follows. A Steiner system $S(v, 4,3)$ is called $(t, \lambda)$-resolvable if its block set $B$ can be partitioned into $r$ subsets $B_{1}, B_{2}, \ldots, B_{r}$ such that $\left(S, B_{i}\right)$ is a $t$-design $T(v, 4, t, \lambda)$ for all $i$. It is clear that

$$
\frac{|B|}{r}=\frac{\left(\begin{array}{l}
v \\
t
\end{array}\right)}{\left(\begin{array}{l}
4 \\
t
\end{array}\right)} \cdot \lambda .
$$


For the case of systems $S(v, 4,3)$ there are two possibilities: $t=1$ or $t=2$. Denote $(t, \lambda)$ resolvable $\operatorname{SQS}(v)$ by $\operatorname{RSQS}(t, \lambda, v)$. If $(t, \lambda)=(1,1)$ such $\operatorname{SQS}(v)$ is also called resolvable, and if $\operatorname{SQS}(v)$ is $((t, 1)$-resolvable simultaneously for $t=1$ and 2 it is also called double resolvable. The first infinite family of double resolvable $\operatorname{SQS}(v)$ for all $v=4^{m}$ was given in [21] (see also [11] and references there). Next, we would like to show that all systems SQS(16) of rank 14 over $\mathbb{F}_{2}$ are resolvable.

\section{REFERENCES}

1. Zinoviev V.A., Zinoviev D.V. Classification of Steiner quadruple systems of order 16 and rank at most thirteen// Problems of Information Transmission. 2004. V. 40. $\mathrm{N}^{\circ}$ 4. P. $48-67$.

2. Hanani H. On quadruple systems// Canad. J. Math. 1960. V. 12. P. 145-157

3. Barrau J.A. On the combinatory problem of Steiner // K. Akad. Wet. Amst. Proc. Sect. Sci. 1908. V. 11. P. 352-360.

4. Mendelsohn N.S., Hung S.H.Y. On the Steiner Systems $S(3,4,14)$ and $S(4,5,15) / /$ Utilitas Math. 1972. V. 1. P. 5-95.

5. Doyen J., Vandensavel M. Non-isomorphic Steiner quadruple systems// Bull. Soc. Math. Belg. 1971. V. 23. P. 393-410.

6. Gibbons P.B., Mathon R., Corneil D.G. Steiner quadruple systems on 16 symbols// In: Proc. 6-th Southeastern Conf. Combinatorics, Graph Theory and Computing, Boca Raton. 1975. Congressus Numerantium XIV (Utilitas Math. Publ. Winnipeg. 1975). P. 345-365.

7. Lindner C.C., Rosa A. There are at least 31021 nonisomorphic Steiner quadruple systems of order 16// Utilitas Math. 1976. V. 10. P. 61-64.

8. Lindner C.C., Rosa A. Steiner quadruple systems - A survay// Discrete Mathematics. 1978. V. 21. P. 147-181.

9. Hartman A., Phelps K.T. Steiner Quadruple Systems// In: Contemporary Design Theory: A Collection of Surveys. Dinitz J.H., Stinson D.R., Eds. John Wiley \& Sons. 1992. Ch. 6. P. 205-240.

10. C.J. Colbourn C.J., J.H. Dinitz J.H. The CRC Handbook of Combinatorial Designs. Boca Raton, FL: CRC Press, 1996.

13. Zinoviev V.A., Zinoviev D.V. Classification of Steiner quadruple systems of order 16 and rank at most thirteen// Proc. Ninth Int. Workshop on Algebraic and Combinatorial Coding Theory. Kranevo, Bulgaria (June 19-25), 2004, P. 399-403.

14. Zinoviev V.A., Zinoviev D. V. Binary extended perfect codes of length 16 by generalized concatenated construction// Problems of Information Transmission. 2002. V. 38. N ${ }^{\circ}$ 4. P. 56-84.

15. Zinoviev V.A., Zinoviev D.V. Binary extended perfect codes of length 16 and rank 14// Problems of Information Transmission. 2004. submitted.

16. Semakov N.V., Zinoviev V.A. Constant weight codes and tactical configurations// Problems of Information Transmission. 1969. V. 5. N 3. P. 29-38. 
17. Zinoviev V.A., Lobstein A. On generalized concatenated constructions of perfect binary nonlinear codes// Problems of Information Transmission. 2000. V. 36. $\mathrm{N}^{\circ}$ 4. P. $59-73$.

18. Doyen J., Hubaut X., Vandensavel M. Ranks of Incidence Matrices of Steiner Triple Systems// Mathem. Zeitschr. 1978. V. 163. P. 251-259.

19. MacWilliams F.J., N.J.A. Sloane N.J.A., The theory of Error Correcting Codes, North-Holland, 1986.

20. Cole F.N., Cummings L.D., White H.S. The complete enumeration of trial systems in 15 elements// Proc. Nat. Acad. Sci. USA. 1917. V. 3. P. 197-199.

21. de Vries H.L. Some Steiner quadruple systems $S(3,4,16)$ such that all 16 derived triple systems $S(2,3,15)$ are isomorphic// Ars Combin. 1987. V. 24A. P. 107-129.

22. Zaitsev G.V., Zinoviev V.A., Semakov N.V. Interrelation of Preparata and Hamming codes and extension of Hamming codes to new double-error-correcting codes, in: "2nd International Symposium on Information Theory, Tsahkadzer, Armenia, USSR, 1971," Akademiai Kiado-Budapest. 1973. P. 257-263.

23. Dickson L.E., Safford F.H. Solution to Problem 8 (Group Theory)// Am. Math. Monthly. 1906. V. 13. P. 150-151.

24. Wallis W.D. On one-factorizations of complete graphs// J. Austral. Math. Soc. 1973. V. 16. P. 167-171.

25. Lindner C.C., Mendelsohn E., Rosa A. On the number of 1-factorizations of the complete graph// J. Combinatorial Theory. B. 1976. V. 20. P. 265-282.

26. Gibbons P.B. Computing techniques for the construction and analysis of block designs// Ph.D Thesis. University of Toronto. 1976.

Zinoviev Victor Alexandrovich

Zinoviev Dmitrii Victorovich

Institute for Information Transmission Problems, Russian Academy of Sciences

zinov@iitp.ru

dzinov@iitp.ru 